\title{
Case series: rheumatological manifestations attributed to exposure to Libby Asbestiform Amphiboles
}

\section{Authors: Roger Diegel, Brad Black, Jean C. Pfau, Tracy McNew, Curtis Noonan, and Raja Flores}

This is an Accepted Manuscript of an article published in Journal of Toxicology and Environmental Health, Part A on June 2018, available online: http:// www.tandfonline.com/10.1080/15287394.2018.1485124.

Diegel, Roger, Brad Black, Jean C. Pfau, Tracy McNew, Curtis Noonan, and Raja Flores. "Case series: rheumatological manifestations attributed to exposure to Libby Asbestiform Amphiboles." Journal of Toxicology and Environmental Health 81, no. 15 (June 2018): 734-747. DOI:10.1080/15287394.2018.1485124. 


\title{
Case Series: Rheumatological Manifestations Attributed to Exposure to Libby Asbestiform Amphiboles
}

\author{
Journal of Toxicology and Environment Health, Part A \\ Roger Diegel ${ }^{1}$, Brad Black$^{2}$, Jean C. Pfau ${ }^{3}$, Tracy McNew ${ }^{2}$, Curtis Noonan ${ }^{4}$, Raja Flores ${ }^{5}$ \\ ${ }^{1}$ Tamarack Medical Clinic, Kalispell MT 59901 \\ ${ }^{2}$ Center for Asbestos Related Diseases, Libby MT 59923 \\ ${ }^{3}$ Montana State University, Bozeman MT 59718 \\ 4University of Montana, Missoula MT 59812 \\ 5Icahn School of Medicine at Mt Sinai, New York NY 10029
}

Running Title: Autoimmune Responses and Libby Amphibole

Corresponding Author:

Jean C. Pfau, Ph.D.

Department of Microbiology and Immunology

Montana State University

960 Technology Blvd

Bozeman MT 59718

406-994-4778

jean.pfau@montana.edu 


\begin{abstract}
An increased risk for Systemic Autoimmune Diseases (SAID) has been reported in Libby, Montana, where extensive exposures to fibrous amphiboles occurred due to mining and use of asbestos-laden vermiculite. In addition, positive antinuclear autoantibody tests are associated with exposure to Libby Asbestiform Amphiboles (LAA) in both humans and mice. Among 6603 subjects who underwent health screening at the Center for Asbestos Related Diseases (CARD, Libby MT), 13.8\% were diagnosed with an autoimmune disease, with prevalence values for the most common SAID being significantly higher than expected in the United States. Among the CARD screening population, serological and clinical profiles are diverse, representing symptoms and autoantibodies reflective of systemic lupus erythematosus (SLE), scleroderma, rheumatoid arthritis and other rheumatic syndromes including Undifferentiated Connective Tissue Disease (UCTD). Based upon screening of medical records by physicians with rheumatology expertise, the evolving nature of rheumatological disease in these patients is often atypical, with mixed diagnostic criteria and with a 1:1 male-to-female ratio. Through the Libby Epidemiology Research Program, cases were identified that illustrate clinical autoimmune outcomes with LAA exposure. Our goal was to better characterize SAID in Libby, MT in order to improve recognition of autoimmune outcomes associated with this exposure. In view of recent discoveries of widespread exposure to fibrous minerals in several areas of the U.S. and globally, it is critical to evaluate rheumatologic manifestations in other cohorts so that screening, surveillance and diagnostic procedures are able to detect and recognize potential autoimmune outcomes of asbestos exposure.
\end{abstract}

Key words: Asbestos, Autoimmune, Autoantibodies, Inhalation Toxicology

Abbreviations: ANA, antinuclear autoantibody; ARD, Asbestos-Related Diseases; ATSDR, Agency for Toxic Substances \& Disease Registry; CARD, Center for Asbestos Related Diseases; CCP, Cyclic citrullinated peptide antibody; CREST, limited cutaneous form of scleroderma; CT, computed tomography; DIP, Distal Interphalangeal Joint; DLCO, Diffusing Capacity of the Lung for $\mathrm{CO}_{2}$; DMARD, Disease Modifying Anti-Rheumatic Drugs; ENA, Extractable Nuclear Antigen antibodies; FVC, Forced Vital Capacity; LAA, Libby Asbestiform Amphiboles; LERP, Libby Epidemiology Research Program; MCP, Metacarpal Phalangeal Joint; PIP, Proximal Interphalangeal Joint; RA, rheumatoid arthritis; RV, Residual Volume; SAID, Systemic autoimmune diseases; SLE, systemic lupus erythematosus; SSc, Systemic Sclerosis; TLC, Total Lung Capacity 


\section{Introduction}

In the town and surrounding areas of Libby, Montana, decades of mining and widespread community use of asbestos-laden vermiculite led to both occupational and environmental exposures to a combination of mineral fibers now termed Libby Asbestiform Amphiboles (LAA). The health outcomes of these exposures that were reported among exposed individuals include both malignant and non-malignant pulmonary diseases, with a severe and progressive pleural fibrosis being the predominant pulmonary outcome (Antao et al 2012; Black, et al. 2014; Bunderson-Schelvan et al, 2011; Larson, et al. 2012; Lockey, et al. 2015; Peipins, et al. 2003; Whitehouse 2004; Whitehouse, et al. 2008). Due to the severity and extent of the asbestos-related disease (ARD) health impacts, Libby, along with the mine, was designated as an EPA Superfund site (Kuntz, et al. 2009; Noonan, et al. 2015; Winters, et al. 2012; 2014.). A nested case-control study of the Libby population screened by the Agency for Toxic Substances and Disease Registry (ATSDR), showed that past LAA exposure was associated with reported systemic autoimmune diseases (SAID) (Noonan, et al. 2006). These observations led to further investigation of immunological responses to LAA exposure in both humans and animal models (Cyphert et al, 2012; 2015; Salazar et al, 2012). A high frequency of positive (and high titer) antinuclear autoantibody (ANA) tests were detected among patients and mice exposed to LAA (Ferro, et al. 2013; Pfau, et al. 2005; 2008; 2009). Several other investigators also reported increased frequencies of ANA and rheumatoid factor among cohorts exposed to asbestos (reviewed in Pfau et al, 2014).

Despite these lines of evidence suggesting an association between asbestos exposure and autoimmune responses, there is not a consensus among experts whether asbestos, particularly amphibole asbestos, needs to be added to the list of triggers for SAID, alongside silica (Noonan and Pfau 2011; Pfau et al 2014; Pfau 2017: Miller, et al. 2012a;2012b). Various 
investigators reported that this uncertainty is based upon the possibility that not all forms of asbestos drive an autoimmune response, and that autoimmune responses are seen more often with amphibole, rather than chrysotile, exposures (Ferro, et al. 2013; Pfau, et al. 2015; Pfau 2017). Historically, chrysotile exposures have been prevalent due to extensive commercial use, but reductions in asbestos mining and product manufacturing are revealing that environmental asbestos exposures are abundant, often contain amphibole fibers, and exert adverse health impacts even at low levels of exposure (Baumann et al. 2013; 2015; Wolfe, et al. 2017; Bayram and Bakan 2014, Buck, et al. 2013, Lockey, et al. 2015, Andujar et al, 2016; Lemen, 2016; Szeinuk, et al. 2017). Therefore, the current study regarding LAA suggests that more research is needed regarding specific mineral fibers that may be triggers for SAID.

The limited epidemiological evidence supporting an association between asbestos exposure and autoimmunity is partially due to the complex case definition for rheumatological syndromes. Conditions that include a complex mixture of autoimmune systems and fail to meet clinical diagnostic criteria may be missed by surveillance and screening efforts. Anecdotally, physicians working with the Libby population perceived a high frequency of rheumatoid conditions that were not being diagnosed because signs and symptoms failed to meet strict diagnostic guidelines. Therefore, two of the objectives of the Libby Epidemiology Research Program (ATSDR) were to a) determine whether a unique rheumatological syndrome existed among LAA-exposed cohort, and b) characterize the population serologically and clinically for rheumatological conditions. The current report focused on this characterization by describing cases of clinical autoimmune outcomes in the LAA-exposed cohort. Our objective was to better understand the complex clinical nature of autoimmune outcomes related to fibrous amphibole exposure. 


\section{Materials \& Methods}

\section{Cohort For Cases Identification}

The Libby Epidemiology Research Program (LERP) cohort was restricted to current or former residents of the cities of Libby and Troy, Montana, ensuring that all subjects had been exposed to LAA. All subjects were 18 years or older, with no restriction regarding sex, smoking history, or medical diagnosis. Recruiting included local newspapers and word-of-mouth, in addition to recruitment through the health screening program at the Center for Asbestos Related Diseases (CARD) in Libby. Following informed consent, all subjects completed extensive medical history questionnaires and provided a blood sample for serum collection by standardized methods. The entire research protocol of the LERP was approved by the Icahn School of Medicine Institutional Review Board, New York, and the Providence Health Care Institutional Review Board, Spokane, Washington. Chart reviews were performed by IRB-approved research team members, and diagnoses were verified by obtaining confirmation of diagnosis or treatment.

The health screening program at the CARD currently contains 6,603 patient records. Of those, $13.8 \%$ have a diagnosis for an autoimmune disease other than diabetes. This database was used to construct Table 1, providing prevalence values for autoimmune diseases in Libby compared to published expected prevalence values in the United States.

\section{Autoantibody Testing}

All serum samples were collected at the CARD clinic by standardized protocols in serum separator tubes, and serum was stored at $-80^{\circ} \mathrm{C}$ until use. All samples were deidentified and given a research number. For patients seen at the CARD, ANA/ENA screening was performed at Pathology Associates Medical Laboratory (PAML) in Spokane, WA. A reflexive ANA screen was performed by ELISA, and if positive, a multiplex (Luminex) bead assay tested for 11 autoantibodies (dsDNA, Smith, Ribosomal P, Chromatin, RNP, SmRNP, Scl- 
70, Centromere B, SSA, SSB and Jo-1). All data were reported by PAML as positive/negative, with titers and values where appropriate. For patients seen by the rheumatologist (RD), testing was conducted at the Mayo Clinic (Mayo Medical Laboratories, Rochester, MN).

In addition, Indirect Immunofluorescence for ANA was performed at Idaho State University (JP) as previously described (Pfau, et al. 2005). Briefly, samples were diluted at 1:40 in phosphate buffered saline, and then placed on wells of commercial ANA slides from ImmunoConcepts (Sacramento, CA). Following incubation, slides were washed and then the secondary antibody (anti-human IgG FITC conjugate) was added to each well. After incubation, washing and cover-slipping, slides were viewed by two blinded readers using a Leica upright fluorescence microscope, and compared against positive controls provided with the slides. Patterns and brightness were evaluated as negative or positive, with positives given a score of 1-4 based on staining intensity. If positive, the samples were titered.

\section{Questionnaire Data}

The CARD used an extensive questionnaire, covering exposure pathways (both occupational and environmental), family history, medical history, and symptoms. The responses were entered into a coded Access database for research access.

\section{Case Descriptions}

Cases were selected by research team physicians (BB, RD) that represent some of the unusual presentations of severe autoimmune disease noted in a concentrated population. A summary of the demographics in this case series is presented in Table 2. All of these cases had relatively high LAA exposure, based upon an extensive exposure matrix (Noonan 2006; Noonan, et al. 2015), with most of them being environmental (not occupational). Most of the cases had lifelong exposures, having grown up in the Libby area, and most developed symptoms in their 30's and 40's, but symptom onset ranged from 20 to 69 years old. The later 
onset cases (\#4 and \#7) were also older when first exposed (Table 2). All of these cases have some form of pulmonary asbestos-related disease. Most are life-long non-smokers.

Although SAID are generally more common in females, in the Libby population, at least half or more of ANA positive subjects were male in randomly selected population subsets in previous studies (Pfau, et al. 2005; 2015). This could reflect the broad, environmental nature of the exposures throughout the community. Based on rheumatology medical histories, many of the cases exhibited atypical presentations of SAID, sometimes in combinations, and with symptoms and diagnoses changing over time. Finally, occurrences of spouses who both have SAID diagnoses were observed in this population (illustrated here as Cases $2 \& 3$ ), an infrequent observation that is suggestive of an exposure-related manifestation of the symptoms.

\section{Case \# 1 - Urticarial Vasculitis}

Currently 58 years old, this patient has been a Libby resident his entire life. He manages a local retail business and is married with grown children. He does not have any family history of arthritis or rheumatism and has been a life-long nonsmoker. The patient has experienced multiple pathways of LAA exposure, including growing up in a home in Libby, MT with vermiculite insulation in the attic and crawl space. His father's primary occupation was logging, which is a documented pathway of LAA exposure due to fiber accumulation in trees, including secondary home exposure as dust is brought home on clothing. He was also exposed to LAA by delivering auto parts to, and taking inventory at, the vermiculite mine site while it was in operation outside of Libby. He played in ore piles as a child and on baseball fields that were surfaced with LAA-containing material. He also reports heavy recreation in the Rainy Creek area for over 20 years. Rainy Creek is the drainage located below the mine site that has significant contamination from mine tailings. He was also a volunteer firefighter for 26 years, 
which might contribute to exposure when buildings, insulated with the vermiculite, burn and release LAA fibers into the ambient air.

This patient was first seen at the Center for Asbestos Related Disease (CARD) for asbestos health screening in mid-2011. He was asymptomatic at the time and diagnosed with smooth, lamellar pleural thickening identified on CT scan. The radiographic description of this type of pleural disease has been described elsewhere (Black, et al. 2014; Szeinuk, et al. 2017). His initial pulmonary functions were within the normal range in 2015 with FVC recorded as $103 \%$ and DLCO $106 \%$.

The patient began developing autoimmune symptoms of recurrent bouts of hives. The hives originally were treated with OTC antihistamines and after a few years, he was seen by a rheumatologist in 2000. Numerous lab tests revealed a low titer ANA at 1:40, but the remainder of the blood tests including rheumatoid factor, $\mathrm{CCP}$, ANCA, complement and cryoglobulins were negative. Patient was instructed to continue with the antihistamines and cimetidine, but did not take them on a daily basis. The hives continued to recur and tended to be around his wrists and ankles, which also caused pain and swelling in his joints. He would have attacks every 4-6 weeks which would last anywhere from two to three days. By 2003, he was beginning to require steroids to control the joint pain and swelling that occurred in his peripheral joints, usually his wrists and ankles. The joints were aspirated and no gout crystals were detected. The aspirations of the joints were done in 2003 and 2004. Because of ongoing symptoms, labs were repeated in 2004 and revealed a negative ANA, negative rheumatoid factor, negative CCP antibody. The patient was diagnosed with urticarial vasculitis and treated with methotrexate with good control of the joint pain and swelling for approximately 10 years. In 2014, he was seen again by the rheumatologist and his vasculitis remained stable. He began developing podagra symptoms and was diagnosed with gout and placed on 
allopurinol and colcrys with good control of pain and swelling. The patient remained on methotrexate with good control of the urticarial vasculitis. Occasionally, he takes prednisone if colchicine does not control the joint pain. The remainder of the review of systems for other autoimmune diseases has been negative.

\section{Case \#2 - Rheumatoid Arthritis}

This patient is currently 57 years old and has been a Libby resident her entire life. She worked in a pharmacy and was a stay-at-home parent to two children. She does not report any family history of autoimmune disease, and she has been a lifelong nonsmoker. Her LAA exposure began as a child when she was raised in a home with vermiculite insulation, and her father, who was a vermiculite mine worker, brought dust home on his clothing. She reports multiple environmental exposures, including gardening in soil contaminated with Libby vermiculite and recreating in the Rainy Creek drainage area over many years.

She was first seen at the CARD in 2009 for asbestos health screening when she was asymptomatic and not diagnosed with ARD. She returned for repeat screening in 2010 at which time she was diagnosed with diffuse pleural thickening on CT scan, and she started to report respiratory symptoms. She was last seen in 2014 at which time she reported having trouble with a chronic cough, wheeze, chest tightness and shortness of breath. Her pulmonary function was normal in 2001 with FVC 100\%, TLC 102\%, DLCO 110\%, and remained quite stable over time.

The patient described rheumatoid arthritis symptoms in 2007, and was diagnosed with rheumatoid arthritis by a rheumatologist in 2009 with joint swelling and fatigue. X-rays revealed no erosions. The patient was on prednisone daily for rheumatoid arthritis and failed many DMARD agents, including methotrexate, plaquenil and sulfasalazine. In 2012, she began treatment with actemra at $4 \mathrm{mg} / \mathrm{kg}$ which was then increased to $8 \mathrm{mg} / \mathrm{kg}$ every 4 weeks IV 
dosing, with methotrexate $25 \mathrm{mg}$ subcutaneous and prednisone $5 \mathrm{mg}$ daily. She did not go into remission. The patient was tried on Orencia, but developed headaches and discontinued the Orencia. The patient began Xeljanz in November 2013 for ongoing synovitis, and a Vectra DA 49 test was conducted in November 2013. The results were reported as greater than 45 , revealing inflammation. The remainder of the review of systems for other autoimmune diseases was negative.

\section{Case \#3 - Rheumatoid Arthritis with Pleurisy}

This patient is currently 60 years old and was a Libby resident his entire life. He worked in various service industries in the area including as a delivery truck driver. He is married with grown children. He does not have any family history of autoimmune disease and has been a lifelong nonsmoker. The patient's LAA exposure occurred throughout his lifetime. His current home was remediated for LAA by EPA two years after he moved in. He also shared a home with a vermiculite mine worker for 3 years and spent significant time participating in recreational activities that may have led to exposure.

This patient was first seen at the CARD for asbestos health screening in 2001. At that time he was asymptomatic with no evidence of asbestos-related disease. In 2007 he was seen at the emergency room on 4 occasions with chest pain and breathing trouble. He was diagnosed at CARD with asbestos-related pleural disease later that year and his symptoms stabilized. His cardiac testing was normal. Most recently he was seen at CARD in mid-2014 when he reported increasing dyspnea on exertion, wheeze, cough, and chest tightness. Initial PFTs in 2007 showed FVC 95\%, TLC 100\%, DLCO 131\%. Despite his increasing symptoms over time, this patient's pulmonary function remained within normal limits and stable from 2007 to 2014. 
The patient in Case \#3 is the husband of patient Case \#2. The patient was diagnosed with rheumatoid arthritis in 2004 with joint swelling in his hands and wrists, and fatigue. He did develop pleurisy and had recurrent bouts from 2008 to 2013. The patient tried several different DMARDs including methotrexate. He did not go into remission and was placed on Arava beginning in July 2011. He did not go into remission. He began treatment with Rituxan in October 2012 with prednisone $5 \mathrm{mg}$ daily, which worked well to control his rheumatoid arthritis symptoms. A Vectra DA done November 2013, was 31. This was conducted while the patient was on Rituxan treatments. The patient's serology in September 2014 was negative ANA, negative CCP. Interestingly, testing did show a moderate positive anti-chromatin test by ELISA, and the patient was positive for myositis antibody PL-12 in 2015.

\section{Case \# 4 - Rheumatic Symptoms with Pleural and Interstitial Disease}

The patient is currently 77 years old and never lived in Libby. He worked operating and renting heavy equipment for most of his career and is married. He reports no family history of autoimmune disease, and has a 12-pack year smoking history. He stopped smoking at age 39. His LAA exposure was from 1974 to 2003 when he worked full-time in a building in Kalispell, MT with vermiculite insulation. He had the vermiculite tested and it was identified to contain $>5 \%$ asbestos content. He reports that this insulation had spilled into his warehouse area, and that he cleaned it up regularly.

The patient was first seen at CARD in mid-2004 when he reported chronic cough, phlegm production and shortness of breath, but was not diagnosed at that time. He was diagnosed with pleural thickening and sub-pleural interstitial fibrosis in 2006. He was last

seen in mid-2015 and reported symptoms that were relatively stable including chest pain. His pulmonary function has shown steady decline over 11 years with FVC of 92\% in 2004 to $47 \%$ in 2015 , TLC from $93 \%$ to $61 \%$, and DLCO from $111 \%$ to $62 \%$. 
The patient developed inflammatory arthritis with swelling in his PIP joints, MCP joints, and wrists in 2009. He also developed Raynaud's phenomenon in 2009 with sun sensitivity and rashes in the sunshine. The patient was seen by a rheumatologist in 2010 . An ANA test was positive at 1:160, central pattern, negative ENA, negative SCL70 antibody, negative anticentromere body, negative CCP, negative rheumatoid factor. The patient was placed on hydroxychloroquine $200 \mathrm{mg}$ b.i.d., which did induce remission of his joint swelling. The patient had repeat serology testing in September 2014, and remained ANA positive.

\section{Case \#5 - CREST and UCTD with Pleural Disease}

This 53 year old man resided in Libby, and nearby Troy, MT, his whole life. He is married and worked in the logging industry throughout his career. He does not report a family history of autoimmune disease and was a lifelong nonsmoker. His LAA exposure began in childhood when he played in ore piles and his family brought ore home from the mine for personal use in landscaping. He also popped vermiculite ore over the heat of the stove to watch it expand.

The patient was first seen at CARD in 2002 and then again in late-2009 when he reported breathing trouble over the last year or two when singing, and occasional sharp pains on the side of his chest. He was diagnosed with bilateral pleural thickening and sub-pleural interstitial changes. He was most recently seen at CARD in early-2014 and reported feeling somewhat better due to weight loss but continues with respiratory symptoms including chronic cough. His pulmonary function remained stable from 2002 to 2014. His most recent PFT in 2017 showed FVC 88\%, FEV1 92\%, and DLCO 117\%. The patient was diagnosed with CREST variant of systemic sclerosis. The symptoms began with arthralgias in 1991 and 1992. The arthralgias continued and the patient saw a rheumatologist. A work up included x-rays of his hands, which were normal, no erosive disease. ANA test of 1:640 with negative SSA, 
negative SSB, negative double stranded DNA antibody, negative anti-Smith antibody, negative RNP antibody, negative rheumatoid factor, negative Lyme. A right forearm nodule was biopsied and revealed a subcutaneous granuloma, most consistent with a rheumatoid nodule. Sub-acute granuloma anulare was also considered in the differential. The patient was placed on hydroxychloroquine without any relief of the arthralgias. The patient was at that time diagnosed with undifferentiated connective tissue disease (UCTD). He started developing severe fatigue in August 1996, and Raynaud's symptoms in July 1998. In 2000 and 2001, he began developing nodules on his forearms, thighs, lower extremities, hands and fingers. Biopsy revealed multicentric reticulohistiocytosis. The symptoms continued, the Raynaud's worsened, and he began developing sclerodactyly in his fingers, loss of pulp in the digits of his fingers and developed sclerodermatous facial features. He was diagnosed with a CREST variant of systemic sclerosis in March 2006. An ANA test in November 2001 had a titer greater than 1:320, and in January 2002, ANA titer was greater than 1:320. No reported ENA values.

\section{Case \#6 - Psoriatic Arthritis and Chronic Lymphocytic Leukemia}

Patient is 58 years old and lived in Libby his whole life. He is married with one child. He worked 9 years in the lumber industry, 22 years in construction and volunteered for the local fire department for over 20 years. He quit work in 2009 and went on disability primarily due to joint problems. Patient reports 26 pack years of smoking history and stopped when he was 48 years old. He also reports a family history of arthritis and psoriasis but is not certain of the exact diagnosis. The patient's LAA exposure began in his early childhood. His father owned and operated a local tire shop and gas station. This business serviced vehicles that were often contaminated with vermiculite dust containing LAA fibers and therefore carried into the home on his father's clothing. There was also a three-year period where the patient's 
older brother, still living at home, worked as a mechanic at the vermiculite mine. The patient reports having spent significant time in the Rainy Creek area, at least 12 years of time playing in or watching games at the downtown ball fields and playing in piles of the vermiculite ore.

The patient was first seen at the CARD in 2010 when he reported that he may be experiencing some dyspnea on exertion and was significantly limited by his arthritis. This is reported to have troubled him over the previous 4-5 years. He had been under treatment for what is described as psoriatic arthritis. He does not report a cough, but does get an occasional, brief pain in the anterior left chest. His screening CT revealed extensive, diffuse pleural thickening, bilaterally. He was last seen by the CARD in 2014. His respiratory symptoms remain minor and stable. His pulmonary function over the years has remained normal with the most recent study showing FVC 93\% FEV 91\% and DLCO 103\%. However, his battle with the psoriatic arthritis and psoriasis has been challenging. Most recently, he was diagnosed with chronic lymphocytic leukemia (CLL).

The patient was diagnosed with psoriasis in 1980 and then developed psoriatic arthritis. He was treated with non-steroidal medications and methotrexate; then in 2011, etanercept was added to the methotrexate. By 2014, etanercept and methotrexate combination became less effective, and etanercept was switched to Simponi in 2015, and he continued with the methotrexate. In the spring of 2015, the patient developed elevated lymphocyte counts and was diagnosed with CLL, and a monoclonal B cell population was seen on flow cytometry. The patient's PAML ANA test was negative with a negative CCP antibody in July 2013. However, subsequent testing revealed a positive ANA with a titer of 1:1280.

\section{Case \#7 - Polymyalgia and Rheumatoid Arthritis with Pleurisy}

Patient is currently 76 years old and was a resident of Libby, MT since the age of 27. He is married with three grown children. He is a retired school teacher and has been self- 
employed as a local rancher. He reports having two siblings with arthritis. He is not certain of the exact diagnoses, but feels that it is a degenerative arthritis. He reports having been a lifelong nonsmoker. The patient experienced multiple pathways of LAA exposure since moving to the area in 1969. His first property in the area had several structures insulated with Libby vermiculite. The existing soil throughout the farm property was heavily contaminated with Libby's vermiculite and LAA. He worked this land for 14 years and was a teacher for over 25 years. While teaching at Libby Middle School, the patient was present during the placement of Libby vermiculite onto the school running track. He coached track for several years and was on or around this track often. He reports hunting several days per year, over at least a 10-year period in the Rainy Creek area. He also reported three separate occasions where he volunteered his time clearing trails (cutting and trimming logs) in the Rainy Creek area.

The patient was first seen at the CARD in 2003. He reported coughing up phlegm each day, having suffered shortness of breath for several years and was experiencing sharp chest pains with a recent episode of pleurisy. He was diagnosed with asbestos-related pleural disease at this time. The patient was last in clinic October of 2015 when he reported still experiencing a chronic cough with phlegm production, dyspnea, wheezing and chest pain. Over time the patient's pulmonary function showed a decline in DLCO (66\%). The patient was diagnosed with polymyalgia rheumatica in 2000 and placed on prednisone. In 2001, he developed swelling in his peripheral joints, hands, wrists, ankles and feet and was diagnosed with seronegative rheumatoid arthritis with negative serology labs, negative ANA, negative rheumatoid factor, negative CCP. X-rays revealed no erosions. He was placed on methotrexate with prednisone, but after 6 months, developed hair loss and oral ulcers, and methotrexate was discontinued. He was placed on leflunomide, did well with leflunomide and 
was able to taper off the prednisone. He was taking leflunomide for 1-l/2 years, but then started developing fluid around his heart and leflunomide was discontinued. The patient then went back on prednisone 20 to $25 \mathrm{mg}$ daily from the early 2000s until 2014. In September 2014, he weaned off prednisone, so he could have a knee replacement. After the knee replacement in 2014, the patient started taking Lipitor, and the arthritis with swelling in his hands, wrists, ankles and feet returned. The patient was placed back on prednisone at $10 \mathrm{mg}$ with tapering doses and $10 \mathrm{mg}$ weekly of methotrexate with good control of his arthritis symptoms. No other autoimmune diseases were present in the patient. The patient had been seen by different rheumatologists from 2000 until 2015, who concurred with the diagnosis of seronegative rheumatoid arthritis. However, subsequent testing showed a positive ANA at 1:2560 titer.

\section{Case \#8 - Rheumatoid Arthritis with Pleural Fibrosis}

Patient is currently 55 years old and was a resident of Libby, MT since the age of 6 . He is married with two grown children. He worked in a retail business for over 30 years. He reports both parents having been diagnosed with ARD. Only one of his three siblings has been seen at the CARD, and he has also been diagnosed with an asbestos-related disease. The patient denies any family history of autoimmune disease. He reports being a lifelong nonsmoker. The patient has experienced multiple pathways of LAA exposure beginning in 1968 when his family moved to Libby, MT. Their first home in the area was remediated for LAA by the EPA after many years of living there. His father was a lumber mill worker for three years, starting when the patient was 9 years old. His father was exposed to dust from contaminated logs and would return home in clothing carrying the LAA fibers as dust. His father also coached sports, spending a lot of time around the ball fields and tracks that were surfaced with LAA-containing material. The patient played most sports in school, placing him 
at these ball fields and tracks. He does recall spending time playing in or around the vermiculite ore piles. As he got older, he rode 4-wheelers in the vermiculite piles and hunted around the entire mine site. His family spent many years collecting and burning firewood from the Rainy Creek area. Wood from this area contains the LAA fibers in the bark (Ward, et al. 2006;2012). His family also gardened with vermiculite ore, which his father brought home.

The patient was first seen at the CARD for asbestos health screening in late 2010. At that time, he reported increasing dyspnea on exertion and that he was experiencing bilateral posterolateral chest discomfort. He did not report a cough or wheeze. On completion of his screening CT, the patient was diagnosed with bilateral pleural thickening in the lower chest posteriorly, which was thin, lamellar and non- calcified. This is consistent with asbestosrelated disease. His pulmonary function test at this time resulted in a FVC of 83\%, DLCO at $116 \%$, TLC at $88 \%$ and RV at $91 \%$ of predicted. When compared to a spirometry assessment completed in 2000, he had lost at least a liter of volume in his FVC.

The patient developed arthralgias and myalgias and was diagnosed with fibromyalgia in $2005 / 2006$. He also developed back pain with some component of inflammatory back symptoms involving his sacroiliac joints. In 2007, he developed synovitis and swelling in his peripheral joints and was diagnosed with rheumatoid arthritis. He was treated with methotrexate in 2007, but did not go into remission. Enbrel was added to methotrexate. The patient was seen by local rheumatologists and was also seen at the Mayo Clinic, and the diagnosis of rheumatoid arthritis was confirmed. The patient did have his regimen switched in 2015 to methotrexate and Humira, with methotrexate $20 \mathrm{mg}$ a week and Humira $40 \mathrm{mg}$ every other week to try to control the synovitis. In 2016, the patient was switched to Actemra $162 \mathrm{mg}$ subcutaneous injection weekly. The patient has had improvement of his synovitis with the Actemra. He is ANA negative. 


\section{Case \#9 - Behçet's Disease, Rheumatoid Arthritis, and Asbestos Related Disease}

This patient is a 50 year old male who grew up in Libby until age 19, moved away and then moved back to the area in 2009. His father worked at the vermiculite mine and would come home with dust from the mine on his clothing. He also played in vermiculite piles, and played baseball on vermiculite-surfaced diamonds as a child. His home was near the railroad tracks where he would play with the ore that fell out of rail cars.

He was in the military but did not work below deck on ships. He drove a log truck for about a year and then worked installing satellite TV and lines where he was exposed to insulation in attics and basements. He is a current smoker with a 29 pack year history, and was first seen at CARD and diagnosed with asbestos-related pleural disease in 2013. He has a chronic cough in the mornings and feels left side chest congestion. He experiences pain in his posterior chest and has been using a Fentanyl patch for pain management.

The patient was diagnosed with Behçet's Disease and rheumatoid arthritis. The patient developed oral and genital ulcers in 1994 with eye inflammation in 1995 and diagnosed with Behçet's disease. The patient developed synovitis in his hands, wrists, ankles and feet and was diagnosed with rheumatoid arthritis in 1995. The patient has been treated with Remicade 500 mg every 4 weeks with good resolution of his symptoms. He also has taken methotrexate $7.5 \mathrm{mg}$ weekly. Prior to Remicade, he had used prednisone. Testing results from PAML from April 2014 showed negative ANA ELISA test, CCP positive at 21, with greater than 20 considered positive. However, indirect immunofluorescence testing gave a positive ANA test at 1:80 titer. The patient also developed pleurisy in 1985.

\section{Case \#10 - Lupus with Pleurisy and Pleural Fibrosis}

The patient is a 53 year old male and lifelong Libby resident. His childhood home was near the railroad tracks, and he spent time playing in areas with vermiculite ore as a child. He 
spent most of his career as a logger. He is a lifelong nonsmoker. He was first seen at the CARD in 2013 and diagnosed with pleural thickening; at that time, he was asymptomatic. At his most recent appointment in June of 2016, he reported cough, wheeze and chest tightness.

The patient developed oral ulcers in 1997. The patient stated he developed problematic joint pain in 2014. He had some arthralgias prior to 2014, including neck pain that developed in 2011. The patient started developing some swelling in his DIP and PIP joints, and started developing pleurisy in 2015. The patient did have a positive ANA test with a positive double stranded DNA antibody in February 2016 and was sent to see a rheumatologist. The patient was diagnosed with lupus with symptoms of joint pain and swelling in his peripheral joints, oral and nasal ulcers and pleurisy. Other lab tests included negative rheumatoid factor and the remaining of the ENA antibodies were negative. X-rays of his hands revealed no erosions, but did show some osteoarthritic changes in his first IP joints and $5^{\text {th }}$ DIP joints. The patient was started on hydroxychloroquine (Plaquinil) $200 \mathrm{mg}$ b.i.d. and the oral ulcers, nasal ulcers, pleurisy and joint pain greatly improved.

\section{Discussion}

Our overall objective with this study was to describe cases of patients whose medical history represented rheumatological clinical profiles of a cohort from Libby, Montana, with exposures to Libby Asbestiform Amphiboles (LAA) in order to characterize and improve recognition of autoimmune outcomes attributed to exposure. Previously, investigators demonstrated elevated frequency and titers of ANA in the Libby population, as well as an increased risk for self-reported SLE, SSc and RA (Noonan, et al. 2006;; Pfau, et al. 2005; 2009). The most common autoantibodies were to dsDNA (specific to both chromatin and histone), Ro-52, and Scl70, with some RNP or Sm (Pfau, et al. 2009). Antibodies associated with RA 
(CCP and rheumatoid factor) are rarely seen in this population (Pfau, et al. 2005; 2009), but the prevalence for RA diagnoses is well above expected levels in the United States general population (Table 1) (Noonan, et al. 2006). CCP- and RF-positive RA is associated with early and rapidly progressive RA, often associated with smoking (Lingampalli, et al. 2018; Shafrin, et al. 2018; Pfau 2012.)

Interestingly, exposure to asbestiform amphiboles also increased the frequency of positive ANA tests in non-autoimmune prone mice and rats (Ferro, et al. 2013; Pfau, et al. 2008; Salazar, et al. 2012). Mice exposed to tremolite, a component of LAA (Meeker, et al. 2003), exhibited positive ANA tests (primarily dsDNA and Ro-52), immune complex deposition in the kidneys and mild glomerular changes suggestive of lupus nephritis (Pfau, et al. 2008). Amosite, also a component of LAA, was also shown to induce ANA in rats (Salazar, et al. 2012). However, exposure to these fibers in two rat models of arthritis failed to significantly elevate indicators of disease (Salazar, et al. 2012).

Noonan, et al (2006) reported a case-control study of self-reported RA, SLE and SSc patients, nested within a medically screened general population cohort in Libby, MT, where extensive exposures to a mixture of asbestiform amphiboles occurred. Data showed more than 4-fold enhanced risk associated with a history of greater LAA exposure via multiple environmental pathways (Noonan, et al. 2006). Since that time, 6603 patients have undergone health screening at the CARD, 387 (6.0\%) having been diagnosed with rheumatoid arthritis, 67 with SLE, 47 with sarcoidosis, and 10 with scleroderma (Table 1). Based upon published prevalence values for these diseases in the United States, all 4 diseases occur in Libby at higher than expected frequency. Prevalence in Libby of other diseases, including multiple sclerosis, ankylosing spondylitis and psoriatic arthritis were not different from expected. 
These data, combined with the animal model studies, present strong evidence that LAA increases the risk for SAID.

However, as physicians (RD, BB) working with LAA-exposed patients, it was noted that there were many cases of a rheumatological clinical profile that were not being diagnosed due to failure to meet the current diagnostic criteria, or that presented with unusual combinations. Symptoms included severe (prolonged) fatigue, Raynaud's phenomenon, rashes, swollen painful joints, and musculoskeletal pain. Although many of the patients also suffer from pulmonary abnormalities indicative of ARD, it is generally not interstitial disease (Antao et al 2012; Peipins, et al. 2003), but rather a thin lamellar pleural thickening (Black, et al. 2014; Szeinuk, et al. 2017). The purpose of this case series was to describe a small sample of people who presented with asbestos-related diseases and autoimmune disease. Pulmonary manifestations are known to occur in patients with SAID. These include various forms of pulmonary pneumonia (infectious, inflammatory and fibrotic), pleural effusions, vasculitis, alveolar hemorrhage, interstitial fibrosis, and obstructive pulmonary disease. Recently, there have been reports of cases of "shrinking lung syndrome" particularly associated with SLE, which manifests as progressive exertional dyspnea, pleuritic chest pain, and restrictive patterns on pulmonary function tests (PFT) (Borrell, et al. 2016; Duron, et al. 2016). However, in the Libby cohort, pulmonary disease (lamellar pleural thickening) is more likely to be associated with asbestos exposure, making the presence of SAID unlikely to be the etiological explanation for the lung disease.

The immunotoxicity of asbestos begins with inflammation, driven by oxidative stress and cytokine production, and the mechanism of autoimmunity may be similar to that proposed for silica, which includes activation of self-reactive B cells by highly activated antigen presenting cells (Cooper, et al. 2008). The effects of asbestos in mice and cell culture 
studies include "frustrated phagocytosis", oxygen radical formation, inflammasome activation, DNA damage, and apoptosis (Bunderson-Shelvan, et al. 2011; Hillegass, et al. 2013; Kodavanti, et al. 2014.) As with silica, mast cells may play a role in initial steps in asbestos autoimmune pathogenesis, since they are activated by asbestos fibers and are involved in the pathogenesis of both allergic and autoimmune diseases (Borelli, et al. 2018; Siebenhaar, et al. 2018.). These early interactions of cells with mineral fibers appear to drive the ultimate disease outcomes, and may be related to specific size, mineralogy, and chemistry of the fibers. In order to work toward therapeutic modalities, further research is needed regarding specific mechanisms driving alterations of self-peptides that make them antigenic, leading to production of autoantibodies.

\section{Conclusions}

In conclusion, the cases described here suggest that there is not one underlying autoimmune disease associated with LAA exposure, but rather a large number of clinical presentations exist among this exposed cohort. They represent severe manifestations of different rheumatological conditions in a highly concentrated population, and this is unusual. In addition, some of the cases demonstrate atypical presentations of SAID, sometimes in combinations, with symptoms and diagnoses changing over time. In many of these patients, it takes years for the final diagnosis to develop, and the evolving nature of the rheumatological symptoms may be quite unusual. Finally, these patients present with pulmonary comorbidities that complicate their clinical picture. Therefore, there are challenges for detection and diagnosis that may mean that the frequency of SAID associated with asbestos exposure may be under-reported. 
Data suggest that exposure to LAA increases the risk of connective tissue disorders and that this requires further study and increased awareness among the rheumatology community. In view of recent discoveries of widespread exposure to similar amphibole fibers in several states (Buck, et al. 2013;Noonan 2017), it is important to understand more about rheumatologic manifestations of exposure such that screening, surveillance and diagnostic procedures are able to detect and recognize autoimmune disease as a potential autoimmune outcome of asbestos exposure.

\section{Acknowledgements}

This work was funded by the Libby Epidemiology Research Program (LERP, CDC/ATSDR TS000099-01). We dedicate this work in the memory of Dr. Stephen Levin, Mount Sinai Medical Center, New York, whose insights and vision spearheaded this work.

\section{Conflicts of Interest}

RD participates in active clinical trials, sponsored by Eli Lilly, Pfizer, AbbVie, R-Pharm US, and Human Genome Science/GlaxoSmithKline. The authors have no other conflicts of interest to disclose. 


\section{References}

Andujar, P., A. Lacourt, P. Borchard, J.C. Pairon, M.C. Jaurand, D. Jean. 2016. Five years update on relationships between malignant pleural mesothelioma and exposure to asbestos and other elongated mineral particles. J Toxicol Environ Health B 19: 151-172.

Antao, V. C., T. C. Larson, and D. K. Horton. 2012. Libby vermiculite exposure and risk of developing asbestos-related lung and pleural diseases. Curr Opin Pulm Med 18: 161167. doi:10.1097/MCP.0b013e32834e897d

Baughman, R. P., S. Field, U. Costabel, R. G. Crystal, D. A. Culver, M. Drent, M. A. Judson, and G. Wolff. 2016. Sarcoidosis in America. Analysis based on health care use. Ann Am Thorac Soc 13: 1244-1252. doi:10.1513/AnnalsATS.201511-7600C

Baumann, F., J. P. Ambrosi, and M. Carbone. 2013. Asbestos is not just asbestos: An unrecognised health hazard. Lancet Oncol 14: 576-578. doi:10.1016/S14702045(13)70257-2

Baumann, F., B. J. Buck, R. V. Metcalf, B. T. McLaurin, D. J. Merkler, and M. Carbone. 2015. The presence of asbestos in the natural environment is likely related to mesothelioma in young individuals and women from Southern Nevada. J Thorac Oncol 10: 731-737. doi:10.1097/JT0.0000000000000506

Bayram, M., and N. D. Bakan. 2014. Environmental exposure to asbestos: From geology to mesothelioma. Curr Opin Pulm Med 20: 301-307. doi:10.1097/MCP.0000000000000053

Black, B., J. Szeinuk, A. C. Whitehouse, S. M. Levin, C. I. Henschke, D. F. Yankelevitz, and R. M. Flores. 2014. Rapid progression of pleural disease due to exposure to Libby amphibole: "Not Your Grandfather's asbestos related disease". Am J Ind Med 57: 11971206. doi:10.1002/ajim.22330

Borelli, V. E. Trevisan, V. Francesca, G. Zabucchi. 2018. The secretory response of rat peritoneal mast cells on exposure to mineral fibers. Int J Environ Res Public Health 15:1-25.

Borrell, H., J. Narvaez, J. J. Alegre, I. Castellvi, F. Mitjavila, M. Aparicio, E. Armengol, M. MolinaMolina, and J. M. Nolla. 2016. Shrinking lung syndrome in systemic lupus erythematosus: A case series and review of the literature. Medicine (Baltimore) 95: e4626. doi:10.1097/MD.0000000000004626

Buck, B. J., D. Goossens, R. V. Metcalf, B. McLaurin, M. Ren, and F. Freudenberger. 2013. Naturally occurring asbestos: Potential for human exposure, Southern Nevada, USA. Soil Sc. Soc Am J 772192-204.

Bunderson-Schelvan, M., J.C. Pfau, R. Crouch, A. Holian. 2011. Non-pulmonary outcomes of asbestos exposure.J Toxicol Environ Health B 14: 122-152. 
Cooper, G.S., K.M. Gilbert, E.L. Greidinger, J.A. James, J.D. Pfau, L. Reinlib, B.C. Richardson and N.R. Rose. 2008. Recent advances and opportunities in research on lupus: Environmental influences and mechanisms of disease. Env Health Perspect 116: 695702.

Cyphert, J.M., D.J. Padilla-Carlin, M.C. Schladweiler, J.H. Shannahan, A. Nyska, U.P. Kodavanti, S.H. Gavett. 2012. Long-term response of rats to single intratracheal exposure of Libby amphible or amosite. J Toxicol Environ Health A 75: 183-200.

Cyphert, J.M., D.J. Carlin, A. Nyska, M.C. Schladweiler, A.D. Ledbetter, J.H. Shannahan, U.P. Kodavanti, S.H. Gavett. 2015. Comparative long-term toxicity of Libby amphibole and amosite asbestosin rats after single or multiple intratracheal exposures. J Toxicol Environ Health A 78: 151-165.

Dilokthornsakul, P., R. J. Valuck, K. V. Nair, J. R. Corboy, R. R. Allen, and J. D. Campbell. 2016. Multiple sclerosis prevalence in the United States commercially insured population. Neurology 86: 1014-1021. doi:10.1212/WNL.0000000000002469

Duron, L., F. Cohen-Aubart, E. Diot, R. Borie, S. Abad, C. Richez, C. Banse, O. Vittecoq, D. Saadoun, J. Haroche, Z. Amoura. 2016. Shrinking lung syndrome associated with systemic lupus erythematosus: A multicenter collaborative study of 15 new cases and a review of the 155 cases in the literature focusing on treatment response and long-term outcomes. Autoimmun Rev 15: 994-1000. doi:10.1016/j.autrev.2016.07.021

Ferro, A., C. N. Zebedeo, C. Davis, K. W. Ng, and J. C. Pfau. 2013. Amphibole, but not chrysotile, asbestos induces anti-nuclear autoantibodies and Il-17 in C57bl/6 mice. J Immunotoxicol 11: 283-290. doi:10.3109/1547691X.2013.847510

Gabriel, S. E., and C. S. Crowson. Epidemiology of, Risk Factors for, and Possible Causes of Rheumatoid Arthritis.UpToDate, https://www.uptodate.com/contents/epidemiologyof-risk-factors-for-and-possible-causes-of-rheumatoid-arthritis.

Hillegass, J.M., J.M Miller, M.B. MacPherson, C.M. Westbom, M. Sayan, J.K. Thompson, S.L. Macura, T. N. Perkins, S.L. Beuschel, V.Alexaeeva, H.I. Pass, C. Steele, B.T. Mossman, and A. Shukla. 2013. Part Fibre Toxicol 10:1-14. doi: 10.1186/1743-8977-10-39.

Jimenez, S. A. Scleroderma: Epidemiology. Medscape, https://emedicine.medscape.com/article/331864-overview\#a5.

Kodavanti, U.P., D. Andrews, M.C. Schladweiler, S.H. Gavett, D.E. Dodd, J.M. Cyphert. 2014. Early and delayed effects of naturally occurring asbestos on serum biomarkers of inflammation and metabolism. J Toxicol Environ Health A 77:1024-1039.

Kuntz, S. W., C. A. Winters, W. G. Hill, C. Weinert, K. Rowse, T. Hernandez, and B. Black. 2009. Rural public health policy models to address an evolving environmental asbestos disaster. Public Health Nurs 26: 70-78. doi:10.1111/j.1525-1446.2008.00755.x 
Larson, T. C., V. C. Antao, F. J. Bove, and C. Cusack. 2012. Association between cumulative fiber exposure and respiratory outcomes among Libby vermiculite workers.

J Occup Environ Med 54: 56-63. doi:10.1097/JOM.0b013e31823c141c

Lemen, R.A. 2016. Mesothelioma from asbestos exposures: Epidemiologic patterns and impact in the United States. J Toxicol Environ Health B 19: 250-265.

Lingampalli, N., J. Sokolove, L.J. Lahey, J.D. Edison, W.R. Gilliland, V. Michael Holers, K.D. Deane, W.H. Robinson. 2018. Combination of anti-citrullinated protein antibodies and rheumatoid factor is associated with increased systemic inflammatory mediators and more rapid progression from preclinical to clinical rheumatoid arthritis. Clin Immunol S1521-6616:30246-8.

Lockey, J. E., K. Dunning, T. J. Hilbert, E. Borton, L. Levin, C. H. Rice, R. T. McKay, R. Shipley, C. A. Meyer, C. Perme, G.K. LeMasters. 2015. HRCT/CT and associated spirometric effects of low Libby amphibole asbestos exposure. J Occup Environ Med 57: 6-13. doi:10.1097/JOM.0000000000000373

Meeker, G. P., A. M. Bern, I. K. Brownfield, H. A. Lowers, S. J. Sutley, T. M. Hoefen, and J. S. Vance. 2003. The Composition and morphology of amphiboles from the Rainy Creek Complex, near Libby, Montana. Am Mineraol 881955-69.

Miller, A. Sjogren Syndrome: Epidemiology. Medscape, https://emedicine.medscape.com/article/332125-overview\#a6.

Miller, F. W., L. Alfredsson, K. H. Costenbader, D. L. Kamen, L. M. Nelson, J. M. Norris, and A. J. De Roos. 2012a. Epidemiology of environmental exposures and human autoimmune diseases: Findings from a National Institute of Environmental Health Sciences Expert Panel Workshop. J Autoimmun 39: 259-271. doi:10.1016/j.jaut.2012.05.002

Miller, F. W., K. M. Pollard, C. G. Parks, D. R. Germolec, P. S. Leung, C. Selmi, M. C. Humble, and N. R. Rose. 2012b. Criteria for environmentally associated autoimmune diseases. J Autoimmun 39: 253-258. doi:10.1016/j.jaut.2012.05.001

Noonan, C. W. 2017. Environmental asbestos exposure and risk of mesothelioma. Ann Transl Med 5: 234. doi:10.21037/atm.2017.03.74

Noonan, C. W. 2006. Exposure matrix development for the Libby cohort. Inhal Toxicol 18: 963-967. doi:10.1080/08958370600835021

Noonan, C. W., K. Conway, E. L. Landguth, T. McNew, L. Linker, J. Pfau, B. Black, J. Szeinuk, and R. Flores. 2015. Multiple pathway asbestos exposure assessment for a superfund community. J Expo Sci Environ Epidemiol 25: 18-25. doi:10.1038/jes.2014.25

Noonan, C. W., and J. C. Pfau. 2011.Asbestos exposure and autoimmune disease. In Encyclopedia of Environmental Health, edited by J. Nriagu, pp.193-203: Elsevier. pp.193-203. 
Noonan, C. W., J. C. Pfau, T. C. Larson, and M. R. Spence. 2006. Nested case-control study of autoimmune disease in an asbestos-exposed population. Environ Health Persp 114: 1243-1247.

Peipins, L. A., M. Lewin, S. Campolucci, J. A. Lybarger, A. Miller, D. Middleton, C. Weis, M. Spence, B. Black, and V. Kapil. 2003. Radiographic abnormalities and exposure to asbestos-contaminated vermiculite in the community of Libby, Montana, USA. Environ Health Persp 111: 1753-1759.

Pfau, J. C. 2017. Immunotoxicity of asbestos. Curr Opin Toxicol 10:1-7.

Pfau, J.C. 2012. Rheumatoid arthritis. In Immunotoxicity, Immune Dysfunction and Chronic Disease, Molecular and Integrative Toxicology, edited by R.R. Dietert and R.W. Luebke, New York, NY: Springer Science \& Business Media, pp. 171-192.

Pfau, J. C., D. J. Blake, and M. J. Fritzler. Autoantibody profiles of an asbestos-exposed population. 2009. In Autoimmunity: Role, Regulation and Disorders, edited by F.L. Vogel and L.F. Zimmermann, . New York, NY: Nova Science Publishers, pp. 245-268

Pfau, J. C., J. J. Sentissi, S. Li, L. Calderon-Garciduenas, J. M. Brown, and D. J. Blake. 2008. Asbestosinduced autoimmunity in C57bl/6 mice. J Immunotoxicol 5: 129-137. doi:10.1080/15476910802085756

Pfau, J. C., J. J. Sentissi, G. Weller, and E. A. Putnam. 2005. Assessment of autoimmune responses associated with asbestos exposure in Libby, Montana, USA. Environ Health Persp 113: 25-30

Pfau, J. C., K. M. Serve, and C. W. Noonan. 2014. Autoimmunity and asbestos exposure. Autoimmune Dis 20141-11. doi:10.1155/2014/782045

Pfau, J. C., K. M. Serve, L. Woods, and C. W. Noonan. 2015. Asbestos exposure and autoimmunity. In Biological Effects of Fibrous and Particulate Substances. Current Topics in Environmental Health and Preventive Medicine: Springer Publications, pp.181-194.

Reveille, J. D. 2011. Epidemiology of spondyloarthritis in North America. Am J Med Sci 341: 284-286. doi:10.1097/MAJ.0b013e31820f8c99

Salazar, K. D., C. B. Copeland, and R. W. Luebke. 2012. Effects of Libby amphibole asbestos exposure on two models of arthritis in the Lewis rat. J Toxicol Environ Health A 75: 351-365. doi:10.1080/15287394.2012.668164

Salazar, K. D., C. B. Copeland, C. E. Wood, J. E. Schmid, and R. W. Luebke. 2012. Evaluation of anti-nuclear antibodies and kidney pathology in Lewis rats following exposure to Libby amphibole asbestos. J Immunotoxicol 10: 329-333.

doi:10.3109/1547691X.2012.747230 
Schur, P. H., and B. H. Hahn. Epidemiology and Pathogenesis of Systemic Lupus Erythematosus. UpToDate, https://www.uptodate.com/contents/epidemiology-and-pathogenesis-ofsystemic-lupus-erythematosus.

Shafrin, J., M.G. Tebeka, K. Price, C. Patel, K. Michaud. 2018. The economic burden of ACPApositive status among patients with rheumatoid arthritis. J Manag Care Spec Pharm 24:4-11.

Siebenhaar, F., F.A. Redegeld, S.C. Bischoff, B.F. Gibbs, M. Maurer. 2018. Mast cells as drivers of disease and therapeutic targets. Trends Immunol 39:151-162. doi: 10.1016/j.it.2017.10.005.

Szeinuk, J., C. W. Noonan, C. I. Henschke, J. Pfau, B. Black, A. Miller, D. F. Yankelevitz, M. Liang, Y. Liu, R. Yip, L. Linker, T. McNew, and R.M. Flores. 2017. Pulmonary abnormalities as a result of exposure to Libby amphibole during childhood and adolescence-the pre-adult latency study (Pals). Am J Ind Med 60: 20-34. doi:10.1002/ajim.22674

Ward, T. J., T. Spear, J. Hart, C. Noonan, A. Holian, M. Getman, and J. S. Webber. 2006. Trees as reservoirs for amphibole fibers in Libby, Montana. Sci Total Environ 367: 460-465. doi:10.1016/j.scitotenv.2006.03.041

Ward, T. J., T. M. Spear, J. F. Hart, J. S. Webber, and M. I. Elashheb. 2012. Amphibole asbestos in tree bark--A review of findings for this inhalational exposure source in Libby, Montana. J Occup Environ Hyg 9: 387-397. doi:10.1080/15459624.2012.682217

Whitehouse, A. C. 2004. Asbestos-related pleural disease due to tremolite associated with progressive loss of lung function: Serial observations in 123 miners, family members, and residents of Libby, Montana. Am J Ind Med 46: 219-225. doi:10.1002/ajim.20053

Whitehouse, A. C., C. B. Black, M. S. Heppe, J. Ruckdeschel, and S. M. Levin. 2008. Environmental exposure to Libby asbestos and mesotheliomas. Am J Ind Med 51: 877880. doi:10.1002/ajim.20620

Winters, C. A., W. G. Hill, K. Rowse, B. Black, S. W. Kuntz, and C. Weinert. 2012. Descriptive analysis of the respiratory health status of persons exposed to Libby amphibole asbestos. Br Med J Open 2. doi:10.1136/bmjopen-2012-001552

Winters, C. A., S. W. Kuntz, C. Weinert, and B. Black. 2014. A case study exploring research communication and engagement in a rural community experiencing an environmental disaster. Appl Environ Educ Commun 13: 213-226.

doi:10.1080/1533015X.2014.970718

Wolfe, C., B. Buck, A. Miller, J. Lockey, C. Weis, D. Weissman, A. Jonesi, P. Ryan. 2017. Int J Hyg Environ Health 220: 1230-1241. 
Table 1

\begin{tabular}{|l|l|l|l|l|}
\hline Disease & $\begin{array}{l}\text { Libby Prevalence: } \\
\text { Number (\%) }\end{array}$ & $\begin{array}{l}\text { Expected } \\
\text { Prevalence }\end{array}$ & $\begin{array}{l}\text { Ratio of } \\
\text { Libby/Expected }\end{array}$ & Source \\
\hline Total of below & $912(13.8 \%)$ & & & $12.5^{\mathrm{b}}$ \\
\hline SLE & $67(1 \%)$ & $0.08 \%$ & $6^{\mathrm{b}}$ & $\begin{array}{l}\text { (Schur and Hahn } \\
\text { 2017) }\end{array}$ \\
\hline RA & $387(6 \%)$ & $1 \%$ & $\begin{array}{l}\text { (Gabriel and Crowson } \\
2016)\end{array}$ \\
\hline SSc & $10(0.2 \%)$ & $0.028 \%$ & $7.1^{\mathrm{b}}$ & (Jimenez 2017) \\
\hline Sarcoidosis & $47(0.7 \%)$ & $0.05 \%$ & $14.0^{\mathrm{b}}$ & $\begin{array}{l}\text { (Baughman, et al. } \\
\text { 2016) }\end{array}$ \\
\hline Ank. Spond. & $14(0.2 \%)$ & $0.2 \%$ & 1 & (Reveille 2011) \\
\hline Psoriatic arthritis & $13(0.2 \%)$ & $0.1 \%$ & 2 & (Reveille 2011) \\
\hline Multiple sclerosis & $15(0.23 \%)$ & $0.15 \%$ & 1.5 & $\begin{array}{l}\text { (Dilokthornsakul, et } \\
\text { al. 2016) }\end{array}$ \\
\hline Sjögrens Synd. & $13(0.2 \%)$ & 0.2 & 1 & (Miller 2017) \\
\hline
\end{tabular}

$\mathrm{a}=$ out of 6603 subjects in screening population

$b=$ statistically significant, $p<0.01$, Chi Square Test with Yates Correction

Abbreviations: SLE (systemic lupus erythematosus), RA (rheumatoid arthritis), SSc (scleroderma), MS

(Multiple Sclerosis), Ank. Spond. (ankylosing spondylitis) 
Table 2

LAA

Age Sex Smoking Exposure Serology

$\begin{array}{llll}\text { Case 1 } & 56 & \mathrm{M} & \text { None } \\ \text { Case 2 } & 55 & \mathrm{~F} & \text { None } \\ \text { Case 3 } & 58 & \mathrm{M} & \text { None } \\ & & & \\ \text { Case 4 } & 75 & \mathrm{M} & \text { Past } \\ \text { Case 5 } & 51 & \mathrm{M} & \text { None }\end{array}$

$\begin{array}{llll}\text { Case } 6 & 56 & \text { M } & \text { Past }\end{array}$

$\begin{array}{llll}\text { Case } 7 & 73 & \mathrm{M} & \text { None }\end{array}$

$\begin{array}{llll}\text { Case } 8 & 53 & \mathrm{M} & \text { None }\end{array}$

$\begin{array}{llll}\text { Case } 9 & 49 & \text { M } & \text { Current }\end{array}$

Case $10 \quad 52 \mathrm{M} \quad$ None
High, Lifelong ANA+

High, Lifelong Neg

Chromatin

High, Lifelong PL-12

High, 1974-

2003

High, Lifelong ANA+

High, Lifelong ANA+ High, 1969-

current

High, Lifelong Neg

(age 6 on)

High, Birth to ANA, age 17, then $\mathrm{CCP}+$ 2009 on.

High, Lifelong ANA+

\section{Symptoms}

(Onset age)

Hives, joint

pain/swelling (38)

Joint swelling,

fatigue (47)

Joint swelling,

fatigue (47) RA

Inflamm Arthritis,

Raynaud's (69) Lupus-like

Arthralgia, $\quad$ CREST, UCTD

fatigue, nodules,

Raynaud's (27)

Joint pain,

swelling (20) Psoriatic arthritis

Polymyalgia, joint

swelling (59)

Arthralgia,

Myalgia, joint

swelling (42)

Ulcers, eye

inflamm, joint

swelling (37)

Ulcers, arthralgia, Lupus

joint swelling (33)
Polymyalgia, RA

RA

Behcet's, RA 